\title{
Particularidades no manejo do diabetes em crianças e idosos
}

\author{
Alessandra S. M. Matheus, ${ }^{1 *}$ Roberta A. Cobas ${ }^{1}$
}

\section{Resumo}

A presente revisão tem por objetivo apresentar e discutir as particularidades no manejo do diabetes em duas populações distintas de pacientes, as crianças e os idosos, através da revisão das principais diretrizes na área, além de revisão da literatura, abordando aspectos importantes de cada grupo etário que tenham impacto nas decisões terapêuticas. O manejo do diabetes em crianças e idosos apresenta alguns pontos em comum. Ambas são populações com características clínicas e psicossociais bastante heterogêneas, nas quais a individualização do tratamento é crucial para a obtenção de sucesso terapêutico. São populações vulneráveis ao risco de hipoglicemia e hiperglicemia extremas e que na grande maioria dos casos o suporte social e familiar é determinante para o tratamento adequado. As metas terapêuticas em crianças preconizadas pela International Society of Pediatric and Adolescent Diabetes (ISPAD) e seguidas pela Sociedade Brasileira de Diabetes são nível de hemoglobina glicada (A1c) $<7,5 \%$ para todos com menos de 18 anos, porém sempre se ressaltando a necessidade de evitar hipoglicemias graves e garantir crescimento e desenvolvimento normais. O tratamento consiste de dieta, atividade física e insulinoterapia exclusiva desde o diagnóstico, para os casos de DM1, ou metformina associada ou não à insulinoterapia, para os casos de diabetes tipo 2. Para idosos, os objetivos e metas de tratamento são variáveis, dependendo da presença de comorbidades, da fragilidade, do deficit cognitivo, da expectativa de vida e do suporte social/familiar do idoso. Sendo assim, as metas de A1c podem variar de 6,5\% a 8,5-9,0\% devendo sempre ser individualizadas. Para a escolha da medicação, devemos considerar o tempo de duração do diabetes, o peso do paciente, sintomas, níveis de glicemia e de HbA1c, o potencial da droga em reduzir a glicemia, seus efeitos extraglicêmicos, seu perfil de segurança, tolerabilidade, posologia e custo..

Descritores: Crianças; Diabetes mellitus; Idoso; Terapêutica.

\section{Abstract \\ Particularities in managing diabetes in children and the elderly}

This review aimed to present and discuss the particularities in managing diabetes in two distinct populations, children and the elderly, through a review of the main guidelines in the area and literature addressing important aspects of each age group which impacts on therapeutic decisions. The management of diabetes in children and the elderly has some points in common. Both populations are very heterogeneous clinical and psychological characteristic in which the individualization of treatment is critical to achieve therapeutic success. Both are
1. Serviço de Diabetes. Hospital Universitário Pedro Ernesto. Universidade do Estado do Rio de Janeiro. Rio de Janeiro, RJ, Brasil.

\author{
*Endereço para correspondência: \\ Boulevard 28 de Setembro, 77 \\ Rio de Janeiro, RJ, Brasil. CEP: 20551-030. \\ E-mail: alessandramatheus79@yahoo.com
}

Revista HUPE, Rio de Janeiro, 2015;14(4):43-49

doi: $10.12957 /$ rhupe.2015.20056

Recebido em 28/11/2014. Aprovado em 09/01/2015.

vulnerable to the risk of hypoglycemia and extreme hyperglycemia and in most cases their social and family support are essential to the suitable treatment. Therapeutic targets in children recommended by the International Society of Pediatric and Adolescent Diabetes (ISPAD) and followed by the Brazilian Society of Diabetes are level of glycated hemoglobin (A1c) $<7.5 \%$ for all under 18 years but always emphasizing the need to avoid severe hypoglycemia and ensure normal development and growth. The treatment is diet, physical activity and insulin since diagnosis in cases of type 1 diabetes. Metformin associated or not with insulin can be used in cases of type 2 diabetes. For the elderly, objectives and treatment goals varies depending on the presence of comorbidity, frailty, cognitive deficit, life expectancy and social/familial support. Thus, A1c targets can range from $6.5 \%$ to $8.5-9.0 \%$ and should always be individualized. The choice of medication must consider the duration of diabetes, patient's weight, presence of symptoms, glycemic control, the potential of the drug in reducing blood glucose, its extra-glycemic effects, its safety profile, tolerability, dosage and cost.

Keywords: Child; Diabetes mellitus; Aged; Therapeutics.

\section{Resumen}

\section{Particularidades en el manejo de la diabetes en niños y adultos mayores}

Esta revisión tiene como objetivo presentar y discutir las particularidades en el manejo de la diabetes en dos poblaciones de pacientes diferentes, los niños y los adultos mayores, a través de la revisión de las principales directrices en esta esfera, además de la revisión de la literatura, abordando los aspectos importantes de cada grupo etáreo que tengan impacto en las decisiones terapéuticas. El control de la diabetes en niños y adultos mayores tiene algunos puntos en común. Ambas son 
poblaciones con características clínicas y psicosociales muy heterogéneas, en las que la individualización del tratamiento es fundamental para alcanzar el éxito terapéutico. Son poblaciones vulnerables expuestas al riesgo de hipoglucemia e hiperglucemia extremas y que en la mayoría de los casos, el apoyo social y familiar es crucial para el tratamiento adecuado. Los objetivos terapéuticos en niños recomendados por la "International Society of Pediatric and Adolescent Diabetes (ISPAD) y seguidos por la "Sociedade Brasileira de Diabetes" son nivel de hemoglobina glicosilada (A1c) $<7,5 \%$ para todo menor de 18 años de edad, pero siempre recalcando la necesidad de evitar hipoglucemias graves y garantizar el crecimiento y desarrollo normales. El tratamiento consiste en una dieta, actividad física y insulinoterapia exclusiva desde el diagnóstico, para los casos

\section{Introdução}

A consideração das particularidades do tratamento do diabetes em crianças e idosos é de extrema importância. Ambas são populações com características bastante heterogêneas, mas nas quais a individualização do tratamento é a base fundamental para o sucesso terapêutico.

O diabetes tipo 1 (DM1) é considerado a segunda doença crônica mais comum na infância e é a principal doença endócrina nesta faixa etária, ${ }^{1}$ correspondendo a cerca de $90 \%$ dos casos de diabetes na infância e adolescência, no entanto menos da metade dos pacientes com DM1 são diagnosticados antes dos 15 anos de idade. ${ }^{2}$ Ainda, com a crescente incidência da obesidade pediátrica, vem crescendo o diagnóstico do diabetes tipo 2 (DM2) também neste grupo etário. ${ }^{3}$ Dados sobre a prevalência de diabetes em menores de 18 anos no Brasil são escassos, entretanto estudo recente realizado no Brasil ressaltou o impacto do custo da doença no sistema público de saúde. ${ }^{4}$

No outro extremo, o estudo multicêntrico brasileiro sobre a prevalência do diabetes no Brasil, realizado na década de 80 , reforçou a importância da idade na prevalência da doença, com incremento de $2,7 \%$ na faixa etária de 30 a 59 anos para $17,4 \%$ na faixa de 60 a 69 anos, caracterizando um aumento de 6,4 vezes. ${ }^{5}$ Dados mais recentes do Ministério da Saúde, através do inquérito epidemiológico VIGITEL/2013, indicam uma prevalência de diabetes de $22 \%$ em indivíduos acima de 65 anos. ${ }^{6}$ Paralelamente, segundo dados do Instituto Brasileiro de Geografia e Estatística (IBGE), a participação relativa de idosos na população brasileira tende a aumentar, estimando que em 2030 indivíduos acima de 60 anos correspondam a 18,62 \% e indivíduos acima de 70 anos a $8,93 \%$ da população.?

Desta forma, concluímos que o contingente de de DM1, o metformina asociada o no a la insulinoterapia, para los casos de diabetes tipo 2. Para los adultos mayores, los objetivos y metas de tratamiento son variables, dependiendo de la presencia de comorbilidades, de la fragilidad, del déficit cognitivo, de la esperanza de vida y del apoyo social/familiar de las personas mayores. Por lo tanto, las metas de A1c pueden variar de 6,5\% a 8,5-9,0\% y deben ser siempre individualizadas. Para elegir la medicación, debemos considerar: el tiempo de duración de la diabetes, el peso del paciente, los síntomas, los niveles de glucemia y de HbA1c, el potencial del fármaco para reducir la glucemia, sus efectos extraglicémicos, su perfil de seguridad, tolerabilidad, posología y costo.

Palabras clave: Niños; Diabetes mellitus; Adultos mayores; Terapéutica.

crianças, adolescentes e idosos portadores de diabetes aumentará progressivamente nas próximas décadas e a qualidade do controle clínico e metabólico dos pacientes impactará, cada vez mais, os programas de saúde pública brasileiros.

O presente capítulo tem por objetivo apresentar e discutir as principais particularidades no manejo do diabetes em duas populações distintas de pacientes -as crianças e adolescentes e os idosos -, por meio da revisão das principais diretrizes na área, além de revisão da literatura abordando aspectos importantes para este manejo.

\section{Manejo do diabetes na infância e adolescência}

A primeira abordagem para o adequado manejo do diabetes na infância e adolescência é a sua classificação. O diabetes tipo 1(DM1) corresponde a cerca de 90\% dos casos nesta faixa etária, ${ }^{2}$ porém a frequência de diabetes tipo 2 (DM2) vem aumentando em paralelo à crescente incidência da obesidade pediátrica. ${ }^{3}$ Da mesma forma, recentemente, foi descrito o surgimento do diabetes duplo ou 1,5 em crianças e adolescentes obesos, que se caracteriza pela presença de diabetes e resistência insulínica associados a níveis adequados de peptídeo C e positividade de autoanticorpos. O sedentarismo, maus hábitos alimentares e a obesidade são os principais fatores que se associam a esses novos tipos de diabetes na infância ${ }^{8}$ (Tabela 1 ).

Geralmente, o DM1 se apresenta na infância com a sintomatologia clássica descrita no adulto, como a poliúria, polidipsia eemagrecimento. No entanto, nesta faixa etária, a polifagia é substituída pela inapetência, recusa alimentar e importante irritabilidade. ${ }^{10} \mathrm{~A}$ balanopostite nos meninos e a candidíase de repetição 
nas meninas também podem ajudar no diagnóstico, assim como a enurese noturna, principalmente quando aparece na criança que já havia obtido o controle esfincteriano. ${ }^{11}$ Em lactentes e pré-escolares, os sintomas de poliúria e polidipsia podem estar ausentes ou passar despercebidos, sendo um importante sinal a perda de peso e a presença de formiga nas fraldas. Dessa forma, merece atenção o diagnóstico de DM1 neste grupo etário através das pistas diagnósticas e sintomas clássicos ao invés da presença de cetoacidose diabética, que está associada a uma maior morbidade e necessidade de doses mais altas de insulina. No estudo Multicêntrico Nacional Brasileiro com DM1 - o BrazDiabSG1 - foi demonstrada uma prevalência de cetoacidose diabética ao diagnóstico de $42 \%$ nesta faixa etária. ${ }^{12}$

A prevalência de outras doenças autoimunes também é marcante no DM1. Dessa forma, o rastreio da doença celíaca e função tireoidiana devem ser realizados imediatamente ao diagnóstico. ${ }^{13}$

O tratamento consiste de dieta, atividade física e insulinoterapia exclusiva desde o diagnóstico para os casos de DM1, ou metformina associada ou não à insulinoterapia, para os casos de diabetes tipo 2 ou duplo. ${ }^{3}$ Em algumas crianças ou adolescentes, o requerimento insulínico pode transitoriamente reduzir após o início da terapia insulínica, o que pode ser definido como período de "lua de mel" ou remissão parcial. Usualmente, as necessidades reduzem para menos de $0,5 \mathrm{UI} / \mathrm{Kg} /$ dia com A1c $<7 \%$, podendo existir níveis glicêmicos estáveis e dentro da faixa da normalidade por semanas ou meses..$^{14,15}$

O desafio no tratamento do diabetes na infância está no fato de que para o alcance das metas glicêmicas ideais esbarra no risco de hipoglicemia, evento adverso da terapia insulínica frequente e comum neste grupo. As crianças mais novas (menores de cinco anos) são as que apresentam maior risco para as sequelas neurológicas (deficit cognitivos) em decorrência de episódios graves de hipoglicemia. ${ }^{16}$

Não somente a hipoglicemia acarreta lesão neurológica na faixa pediátrica, mas, atualmente, estudos demonstraram que a hiperglicemia crônica em crianças com menos de cinco anos ocasiona disfunção da massa cerebral branca devido a uma deficiente mielinização. ${ }^{17}$ Dessa forma, recentemente, a Associação Americana de Diabetes (ADA) mudou os alvos de A1c, que antigamente eram divididos por faixas etárias, para $<7,5 \%$ para todos com menos de 18 anos em concordância com a International Society of Pediatric and Adolescent Diabetes (ISPAD). ${ }^{13,18}$ No entanto, deve ser enfatizado que a individualização das metas ainda é soberana, objetivando o alcance do melhor controle possível com

Tabela 1. Principais diferenças entre os tipos de diabetes na infância.

\begin{tabular}{|c|c|c|c|}
\hline & Diabetes duplo & DM1 & DM2 \\
\hline CURSO CLÍNICO & Variável & Rápido dx com sintomas & Muitos meses \\
\hline Idade início & $\begin{array}{l}\text { Infância e adolescência ++ } \\
\text { Adulto + }\end{array}$ & $\begin{array}{l}\text { Infância e adolescência +++ } \\
\text { Adulto + }\end{array}$ & $\begin{array}{l}\text { Infância + } \\
\text { Adolescência ++ } \\
\text { Adulto +++ }\end{array}$ \\
\hline Peso & Sobrepeso/obesidade & Magro (sobrepeso) & Sobrepeso/Obesidade \\
\hline Ambiente & Estilo de vida & Dieta, vírus & Estilo de vida \\
\hline HFAM & DM1 e/ou DM2 & $5 \%$ DM1 & $\begin{array}{l}20 \% \text { DM } 2 \text { - Pelo menos } 1 \\
\text { parente com DM2 }\end{array}$ \\
\hline Resistência insulínica & ++ & $-/+$ & +++ \\
\hline Peptídeo C & + & $\begin{array}{l}\text { Pode estar reduzido ao } \\
\text { diagnóstico }\end{array}$ & +++ \\
\hline Autoanticorpos & + & $85 \%$ & $15 \%$ \\
\hline Marcadores inflamatórios & ++ & + & +++ \\
\hline
\end{tabular}

DM1 - Diabetes tipo 1; DM2 - Diabetes tipo 2; dx - diagnóstico; HFAM - história familiar (adaptado deª). 


\section{Artigo de revisão}

menores riscos de hipo ou hiperglicemias graves, assim como a adequação do crescimento e desenvolvimento puberal. As metas para glicemias em jejum e pré-prandiais, pós-prandiais, ao deitar e na madrugada são 70-145 $\mathrm{mg} / \mathrm{dL}, 90-180 \mathrm{mg} / \mathrm{dL}, 120-180 \mathrm{mg} / \mathrm{dL}$ e 80-162 mg/dL, respectivamente, de acordo com a ISPAD. ${ }^{18}$

Por tratar-se de uma doença crônica manifestada na infância, a educação e orientação no tratamento devem ser fortemente enfatizadas, principalmente com os pais, mas também com outros cuidadores (avós e vizinhos) e com os professores nas escolas, devendo ser multidisciplinar (sendo a equipe minimamente composta por nutricionista, enfermeira e médico), contínua e frequente para ser efetiva. ${ }^{19-21}$

Outros aspectos também precisam ser ressaltados, como a orientação da família e da escola para os dias de doença, o calendário vacinal adequado e a monitorização do crescimento e desenvolvimento puberal, além de rigoroso acompanhamento nutricional e orientação para a prática de atividade física. Dessa forma, o tratamento do diabetes na infância é complexo, devendo ser abordado de forma multidisciplinar.

\section{Manejo do diabetes em pacientes idosos}

Idosos portadores de diabetes são uma população particularmente suscetível ao desenvolvimento de complicações micro ou macrovasculares e muitas vezes agregam múltiplos fatores de risco e comorbidades. Entretanto, além das complicações tradicionalmente reconhecidas, o diabetes vem sendo relacionado a um declínio excessivo da função cognitiva e depressão. Ambas as condições podem afetar a adesão ao tratamento e resultar em risco de complicações agudas (hipoglicemia ou descompensação da glicemia) e crônicas.

Como resultado dessa tendência, diretrizes específicas para o manejo do diabetes nesta faixa etária vêm sendo publicadas, entre elas o European Diabetes Working Party for Older People 2011 - EDWPOP -,22 Diabetes in older adults: A consensus report American Diabetes Association (ADA) e American Geratrics Society 2012,23 as Diretrizes da Sociedade Brasileira de Diabetes 2013-2014² e, mais recentemente, o Global Guideline - International Diabetes Federation (IDF): Managing Older People with Type 2 Diabetes. ${ }^{24}$ As considerações discutidas neste texto são baseadas nas principais recomendações destas diretrizes.

O tratamento do diabetes nos idosos deve ser multifatorial, especialmente focado em prevenção de incapacidade funcional, complicações crônicas e/ou agudas e disfunção cognitiva. Para isso, uma equipe multiprofissional capacitada é indispensável.

Esse é um grupo de pacientes em que a individualização do tratamento é de extrema importância. Então, as decisões terapêuticas devem ser focadas na relação risco-benefício para cada paciente de forma individual. As principais questões a serem consideradas para as decisões terapêuticas são:1) definir objetivos do tratamento, 2) definir as metas para atingir os objetivos, 3) propor o esquema terapêutico apropriado.

Na definição dos objetivos do tratamento devemos considerar: a estratificação de risco de hipoglicemia, hiperglicemia e suas consequências de quedas e de eventos adversos; o estado funcional dos idosos, o custo do tratamento, a presença de comorbidades/fragilidade e a expectativa de vida. ${ }^{24}$ Algumas outras questões de mesma importância devem ser consideradas. Indivíduos diabéticos idosos têm maior chance de apresentar síndromes geriátricas comuns, como polifarmácia (que interfere na adesão ao tratamento, além do risco de interação medicamentosa), depressão, disfunção cognitiva, incontinência urinária e risco de dor crônica. ${ }^{25}$ A ADA recomenda rastreamento periódico de depressão através da escala de depressão geriátrica (Geriatric Depression Scale). ${ }^{23,26}$ Em relação à função cognitiva, a ADA ressalta que muitos casos não são diagnosticados e que por isso deve ser realizado rastreamento periódico. ${ }^{5}$ O EDWPOP preconiza rastrear disfunção cognitiva em maiores de 70 anos em intervalos regulares utilizandose, por exemplo, o Minimental State Examination. ${ }^{22,27}$

Desta forma, os principais objetivos do tratamento são: eliminar sintomas da doença (hiper ou hipoglicemia), evitar instabilidade glicêmica, melhorar a qualidade de vida, evitar complicações agudas (com risco de desidratação e evolução para estado hiperosmolar), prevenir ou retardar complicações crônicas e reduzir mortalidade.

Uma vez definidos os objetivos, devemos estabelecer metas, ou seja, quais valores de glicemia e hemoglobina glicada (A1c) que refletem o ponto de equilíbrio entre risco de hipoglicemia e descompensações hiperglicêmicas. Para idosos, algumas considerações especiais na definição das metas são destacadas nas diretrizes. Segundo a $\mathrm{SBD}^{28}$ os níveis de A1c em adultos devem ser mantidos nos valores mais baixos possíveis, sem aumentar desnecessariamente o risco de hipoglicemia, sobretudo em pacientes com doença cardiovascular e em uso de insulina. Para a ADA, idosos com boa condição funcional e função cognitiva e significativa expectativa de vida devem ser tratados buscando os alvos determinados para adultos jovens (A1c < 7\%). ${ }^{29}$ 
Níveis de A1c menores $(6,0-6,5 \%)$ podem ser considerados em pacientes com menor duração da doença, longa expectativa de vida e sem doença cardiovascular significativa. Níveis de A1c maiores (7,5-8,0\% ou ainda mais altos) podem ser considerados em pacientes com história de hipoglicemia grave, limitada expectativa de vida, complicações crônicas avançadas, comorbidades importantes, com dificuldade de obtenção das metas apesar de tratamento intensivo. ${ }^{29}$ Para idosos com complicações avançadas, comorbidade grave e/ ou expectativa de vida menor que cinco anos, níveis de A1c entre 8,0 e 9,0\% seriam aceitáveis. ${ }^{23}$ Entretanto, hiperglicemia que cause sintomas ou risco de complicações agudas deve ser evitada em todos os pacientes. ${ }^{25} \mathrm{O}$ EDWPOP preconiza como metas níveis de HbA1c entre 7,0 e 7,5\% para idosos sem comorbidades e níveis mais elevados (HbA1c 7,6-8,5\%) para idosos frágeis pelo alto risco de hipoglicemia e descompensação metabólica. ${ }^{22}$ Segundo a IDF, para idosos com bom estado funcional e maior expectativa de vida, podemos almejar níveis de HbA1c entre 7,0 e 7,5\%; para aqueles funcionalmente dependentes (fragilidade e/ou demênica), níveis até 8,5\% são aceitáveis, e para aqueles em fim de vida, a prioridade é evitar sintomas, sem metas definidas de HbA1c. ${ }^{24}$

Uma vez definidas as metas, devemos estabelecer o melhor esquema terapêutico para atingi-las. Características individuais devem ser consideradas, como perda da independência pessoal e social (necessidade de suporte/cuidadores), diminuição da memória (esquecimento de refeições, do uso dos medicamentos, da monitorização), deficiência em solucionar problemas, tomar decisões e julgar (dificuldade em reconhecer e prontamente tratar episódios de hipoglicemia e hiperglicemia).

Para a escolha da medicação, devemos considerar o tempo de duração do diabetes e as características clínicas do paciente, como peso, sintomas, níveis de glicemia de jejum e pós-prandial e HbA1c, o potendial da droga em reduzir a glicemia, seus efeitos extraglicêmicos, seu perfil de segurança, tolerabilidade, posologia e custo (Tabela 2). As drogas devem ser iniciadas na menor dose e devemos acompanhar a progressão da dose considerando as metas terapêuticas estabelecidas e/ou o desenvolvimento de eventos adversos.

Devemos antecipar dificuldades como, por exemplo, dificuldade de deglutição, assegurar a compreen-

Tabela 2. Perfil das diferentes intervenções terapêuticas para o manejo do diabetes. ${ }^{24,29}$

\begin{tabular}{|c|c|c|c|}
\hline Intervenção & $\begin{array}{c}\text { Redução da A1c como } \\
\text { monoterapia }\end{array}$ & Vantagens & Desvantagens \\
\hline $\begin{array}{l}\text { Redução de peso e } \\
\text { atividade física }\end{array}$ & $1,0-2,0 \%$ & Inúmeras & Insuficiente para a maioria \\
\hline Metformina & $1,0-2,0 \%$ & Peso, baixo risco hipoglicemia & Efeitos gastrointestinais \\
\hline Sulfonilureias & $1,0-2,0 \%$ & Efeito rápido & Ganho de peso, hipoglicemia \\
\hline Insulina & $1,5-3,5 \%$ & $\begin{array}{l}\text { Sem limite de dose, efeito } \\
\text { rápido }\end{array}$ & $\begin{array}{l}\text { Ganho de peso, hipoglicemia, } \\
\text { custo (análogo), logística }\end{array}$ \\
\hline TZDs (pioglitazona) & $0,5-1,4 \%$ & Benefício do perfil lipídico & $\begin{array}{l}\text { Retenção de fluido, ICC, } \\
\text { ganho de peso, fratura, alto } \\
\text { custo }\end{array}$ \\
\hline $\begin{array}{l}\text { Agonista de GLP-1 (exenatide, } \\
\text { liraglutide, lixisenatide) }\end{array}$ & $0,5-1,0 \%$ & Perda de peso & $\begin{array}{l}\text { Injetável, efeitos gastroin- } \\
\text { testinais, segurança descon- } \\
\text { hecida a longo prazo, alto } \\
\text { custo }\end{array}$ \\
\hline Inibidores de DPP-IV & $0,5-0,8 \%$ & Peso $\leftrightarrow$ & $\begin{array}{l}\text { Segurança desconhecida a } \\
\text { longo prazo, alto custo }\end{array}$ \\
\hline $\begin{array}{l}\text { Glinidas (repaglinida, } \\
\text { nateglinida) }\end{array}$ & $0,5-1,5 \%$ & Efeito rápido & $\begin{array}{l}\text { Ganho de peso, posologia } \\
\text { (3x/dia), hipoglicemia, custo }\end{array}$ \\
\hline Acarbose & $0,5-0,8 \%$ & Peso, baixo risco hipoglicemia & $\begin{array}{l}\text { Efeitos gastrointestinais, } \\
\text { posologia ( } 3 x / \text { dia), custo }\end{array}$ \\
\hline Inibidores do SGLT-2 & & Peso, baixo risco hipoglicemia & $\begin{array}{l}\text { Infecção genital e ITU, hipo- } \\
\text { volemia, hipotensão postural }\end{array}$ \\
\hline
\end{tabular}


são da prescrição, reforçar educação em diabetes de forma individualizada, considerando capacidade de compreensão e memória, deficit visual e/ou auditivo, habilidade manual e condição social e compartilhar a decisão terapêutica. ${ }^{24}$

O EDWPOP recomenda, para idosos não obesos, os secretagogos de insulina (sulfonilureias ou glinidas) ou metformina e, para idosos com obesidade, a metformina. Quando for necessária associação de drogas, para aqueles com peso normal ou sobrepeso, metformina + sulfonilureias ou inibidores da DPP-4. Recomenda-se evitar a glibenclamida em maiores de 70 anos. Com relação às insulinas, podemos considerar as pré-misturas e canetas para maior comodidade na aplicação e menor risco de erro de dose, e ainda considerar análogos de longa ação em vez de insulina humana NPH. ${ }^{22}$ Para a ADA, a metformina é droga de primeira linha para a maioria dos idosos pelo baixo risco de hipoglicemia na ausência de contraindicações, particularmente disfunção renal, e em idosos frágeis devemos considerar seus efeitos colaterais. Glinidas são boas opções para idosos com hábitos alimentares irregulares. ${ }^{23}$

\section{Conclusão}

Para crianças e idosos portadores de diabetes, as metas terapêuticas e a escolha do esquema terapêutico devem ser individualizadas e determinadas pelo objetivo principal do tratamento e condição clínica e suporte social do paciente. Para crianças, devemos garantir um controle metabólico adequado, evitando risco de hipoglicemias, além de assegurar crescimento e desenvolvimento normais. Para idosos, devem ser considerados a duração do diabetes, idade e expectativa de vida, presença de comorbidades, risco de hipoglicemias especialmente se assintomáticas e fatores individuais de cada paciente. A educação em diabetes voltada para o paciente, familiares ou cuidadores, talvez seja a principal meta a ser alcançada para prevenir complicações agudas e crônicas da doença.

\section{Referências}

1. Centers for Disease Control and Prevention. National Diabetes Statistics Report: Estimates of Diabetes and Its Burden in the United States, 2014. Atlanta, GA: U.S. Department of Health and Human Services; 2014 [cited 2015 nov 28]. Available from: http://www.cdc.gov/diabetes/pubs/statsreport14/national-diabetes-report-web.pdf

2. Thunander M, Petersson C, Jonzon K, et al. Incidence of type 1 and type 2 diabetes in adults and children in Kronoberg, Sweden. Diabetes Res Clin Pract. 2008 Nov;82(2):247-55.

3. Zeitler P. Approach to the obese adolescent with new-onset diabetes. J Clin Endocrinol Metab. 2010 Dec;95(12):5163-70.
4. Cobas RA, Ferraz MB, Matheus AS, et al. The cost of type 1 diabetes: a nationwide multicentre study in Brazil. Bull World Health Organ. 2013 Jun 1;91(6):434-40. doi: 10.2471/ BLT.12.110387. Epub 2013 Apr 4.

5. Malerbi DA, Franco LJ. Multicenter study of the prevalence of diabetes mellitus and impaired glucose tolerance in the urban Brazilian population aged 30-69 yr. The Brazilian Cooperative Group on the Study of Diabetes Prevalence. Diabetes Care. 1992 Nov;15(11):1509-16.

6. Brasil. Ministério da Saúde. Secretaria de Vigilância em Saúde. Vigitel Brasil 2013: vigilância de fatores de risco e proteção para doenças crônicas por inquérito telefônico /Ministério da Saúde, Secretaria de Vigilância em Saúde. Brasília: Ministério da Saúde, 2014 [Acesso em: 28 nov 2015]. Disponível em: https://biavati.files.wordpress.com/2014/05/vigitel-2013.pdf

7. Instituto Brasileiro de Geografia e Estatística. Projeção da população do Brasil e das Unidades da Federação;2015 [acesso em dec 2015 20]. Disponível em: http://www.ibge.gov.br/apps/ populacao/projecao/

8. Pozzilli P, Guglielmi C. Double diabetes: a mixture of type 1 and type 2 diabetes in youth. Endocr Dev. 2009;14:151-66.

9. Pozzilli P, Buzzetti R. A new expression of diabetes: double diabetes. Trends Endocrinol Metab. 2007 Mar;18(2):52-7.

10. Kaufman FR. Diabetes in children and adolescents. Areas of controversy. Medical Clinics of North America. 1998;82(4):721-38.

11. Calliari LE. Tratamento ambulatorial do diabetes mellitus tipo 1. In: Monte LAC, ed. Endocrinologia para o Pediatra. São Paulo: Atheneu;1998. p. 82-7.

12. Gomes MB, Mattos Matheus AS, Calliari LE, et al. Economic status and clinical care in young type 1 diabetes patients: a nationwide multicenter study in Brazil. Acta Diabetol. 2013 Oct;50(5):743-52.

13. Siminerio LM, Albanese-O'Neill A, Chiang JL, et al. Care of Young Children With Diabetes in the Child Care Setting: A Position Statement of the American Diabetes Association. Diabetes Care. 2014 Oct;37(10):2834-42.

14. Sochett EB, Daneman D, Clarson C, et al. Factors affecting and patterns of residual insulin secretion during the first year of type 1 (insulin-dependent) diabetes mellitus in children. Diabetologia. 1987 Jul;30(7):453-9.

15. Hathout EH, Hartwick N, Fagoaga OR, et al. Clinical, autoimmune, and HLA characteristics of children diagnosed with type 1 diabetes before 5 years of age. Pediatrics. 2003 Apr;111(4 Pt 1):860-3.

16. Seaquist ER, Anderson J, Childs B, et al. Hypoglycemia and diabetes: a report of a workgroup of the American Diabetes Association and the Endocrine Society. Diabetes Care. 2013 May;36(5):1384-95.

17. Marzelli MJ, Mazaika PK, Barnea-Goraly N, et al. Neuroanatomical correlates of dysglycemia in young children with type 1 diabetes. Diabetes. 2014 Jan;63(1):343-53.

18. Rewers MJ, Pillay K, de Beaufort C, et al. Assessment and monitoring of glycemic control in children and adolescents with diabetes. Pediatr Diabetes. 2014 Sep;15 Suppl 20:102-14.

19. Funnell MM, Brown TL, Childs BP, et al. National standards for diabetes self-management education. Diabetes Care. 2007 Jun;30(6):1630-7.

20. Lange K, Sassmann H, von Schutz W, et al. Prerequisites for age-appropriate education in type 1 diabetes: a model programme for paediatric diabetes education in Germany. Pediatr Diabetes. 2007 Oct;8 Suppl 6:63-71.

21. von Sengbusch S, Muller-Godeffroy E, Hager S. Mobile dia- 
Alessandra S. M. Matheus, Roberta A. Cobas • Particularidades no manejo do diabetes em crianças e idosos

betes education and care: intervention for children and young people with type 1 diabetes in rural areas of northern Germany. Diabetic Medicine. 2005;23:122-7.

22. Group EDWpfoPER. Clinical Guidelines for type 2 Diabetes Mellitus (EDWPOP). Diabetes and Metabolism. 2011;37(3):S28-S38.

23. Sue Kirkman M, Briscoe VJ, Clark N, et al. Diabetes in older adults: a consensus report. J Am Geriatr Soc. 2012 Dec;60(12):2342-56.

24. International Diabetes Federation. IDF global guideline for managing older people with type 2 diabetes; 2013; [Access on: 2015 nov 21]. Available from: http://www.idf.org/sites/default/ files/IDF-Guideline-for-older-people-T2D.pdf

25. Chen LK, Chen YM, Lin MH, et al. Care of elderly patients with diabetes mellitus: a focus on frailty. Ageing Res Rev. 2010 Nov;9 Suppl 1:S18-22.
26. Paradela EM, Lourenco RA, Veras RP. Validation of geriatric depression scale in a general outpatient clinic. Rev Saude Publica. 2005 Dec;39(6):918-23.

27. Brucki SM, Nitrini R, Caramelli P, et al. Suggestions for utilization of the mini-mental state examination in Brazil]. Arq Neuropsiquiatr. 2003 Sep;61(3B):777-81.

28. Tratamento de pacientes idosos com diabetes. In: Sociedade Brasileira de Diabetes, ed. Diretrizes da Sociedade Brasileira de Diabetes 2013-2014. São Paulo: GEN; 2013 [Acesso em: 20 nov 2015]. Disponível em: http://www.diabetes.org.br/images/ pdf/diretrizes-sbd.pdf

29. Inzucchi SE, Bergenstal RM, Buse JB, et al. Management of hyperglycemia in type 2 diabetes: a patient-centered approach: position statement of the American Diabetes Association (ADA) and the European Association for the Study of Diabetes (EASD). Diabetes Care. 2012 Jun;35(6):1364-79. 